\title{
Quark Potential in a Quark-Meson Plasma
}

\author{
Chengfu $\mathrm{Mu}$ and Pengfei Zhuang \\ Physics Department, Tsinghua University, Beijing 100084, China
}

\begin{abstract}
We investigate quark potential by considering meson exchanges in the two flavor Nambu-JonaLasinio model at finite temperature and density. There are two kinds of oscillations in the chiral restoration phase, one is the Friedel oscillation due to the sharp quark Fermi surface at high density, and the other is the Yukawa oscillation driven by the complex meson poles at high temperature. The quark-meson plasma is strongly coupled in the temperature region $1 \leq T / T_{c} \lesssim 3$ with $T_{c}$ being the critical temperature of chiral phase transition. The maximum coupling in this region is located at the critical point.
\end{abstract}

PACS numbers: 11.30.Rd, 11.10.Wx, 25.75.Nq

\section{INTRODUCTION}

It is widely accepted that there are two QCD phase transitions at finite temperature and density, one is the deconfinement and the other is the chiral symmetry restoration. The only possible way to realize the phase transitions in laboratories is through high energy nuclear collisions. From the experimentally observed anisotropic collective flow at RHIC which is characterized by the perfect fluid, the surviving resonant states of quarks and gluons above the critical temperature $T_{c}$ of the phase transitions calculated in lattice QCD and effective models, and the significant difference between the lattice calculated thermodynamic functions and the corresponding Stefan-Boltzmann limits at extremely high temperature, the created new state of matter at RHIC is a strongly coupled quark-gluon plasma 1]. With the free energy of a pair of heavy quarks calculated in lattice QCD [2], the heavy quark potential can be extracted. The depth of the potential well decreases with increasing temperature and the dissociation temperature of the bound state $J / \Psi$ is about 2.7 $T_{c}$ [3].

From Yukawa's idea that nucleon-nucleon scattering is through boson exchanges, the static nucleon potential is a Fourier transformation of the boson propagator at momentum $K_{\mu}=\left(k_{0}=0, \mathbf{k}\right)$,

$$
V(r)=\int \frac{d^{3} \mathbf{k}}{(2 \pi)^{3}} e^{i \mathbf{k} \cdot \mathbf{r}} U\left(0, k^{2}\right) .
$$

The Yukawa potential is widely discussed in nuclear matter and recently extended to quark matter, especially, its two kinds of oscillations, the Friedel oscillation [4, 5, 6, 7, 8, 9] induced by the sharp Fermi surface at high density and the Yukawa oscillation [8, 10] resulted from the complex poles of the exchanged bosons, are frequently emphasized.

To understand the quark behavior in strongly coupled quark matter with quarks, gluons and their bound states as constituents, we investigate in this paper the light quark potential through meson exchanges in a quarkmeson plasma controlled by chiral dynamics. By calculating the quark potential around the chiral phase transition, we hope to see the effect of chiral symmetry restora- tion on the quark properties, to extract the information on the temperature region of strong coupling, and to know where the maximum coupling is.

In our calculation we construct mesons in quark matter in the frame of Nambu-Jona-Lasinio [11] (NJL) model at quark level [12]. The NJL model is inspired from the electron superconductivity. It describes well the chiral properties in vacuum and in hot and dense medium. Above the critical temperature, there exist hadronic excitations with small widths [13, 14]. The spatial dependence of static meson correlation functions in the model was investigated at finite temperature [15] and density [16]. In recent years, the model is successfully used to study color superconductivity at moderate baryon density [17] and pion superfluidity at finite isospin density [18]. To understand the results of lattice QCD thermodynamics in terms of quasiparticle degrees of freedom, the model is recently extended to include Polyakov loop dynamics (PNJL) [19, 20].

To define the NJL model completely as an effective theory, a regularization scheme must be specified to deal with the important integrals that occur. A regularization scheme specifies a length scale for the theory, which is normally expressed as a cutoff $\Lambda$ on the quark momentum and meson momentum. From the uncertainty principle, the length scale in the NJL model is $R \sim 1 / \Lambda$. Taking the standard cutoff value $\Lambda \sim 600 \mathrm{MeV}$ [12], we have $R \sim 1 / 3 \mathrm{fm}$. This means that, the quark potential calculated in the NJL model might be reasonable in the region of $r>1 / 3 \mathrm{fm}$. In the short range the model is probably not applicable. While we will take a covariant Pauli-Villars regularization scheme which allows us to do the momentum integrations in the whole region without an explicit cutoff, we still focus only on the long range behavior of the quark potential with $r>1 / 3 \mathrm{fm}$.

The paper is organized as follows. We review the analytic properties of meson polarization functions in the Pauli-Villars regularization scheme and determine the meson poles in the complex momentum plane in Section II. In Section III we show the quark potential at finite temperature and density and analyze the effect of chiral symmetry restoration. The temperature region of strongly coupled quark-meson plasma and the position 
corresponding to the maximum coupling will be discussed in this section. We conclude in Section [IV]

\section{QUARK POTENTIAL}

The flavor SU(2) NJL model is defined through the lagrangian density

$$
\mathcal{L}=\bar{\psi}\left(i \gamma^{\mu} \partial_{\mu}-m_{0}+\mu \gamma_{0}\right) \psi+G\left[(\bar{\psi} \psi)^{2}+\left(\bar{\psi} i \gamma_{5} \tau \psi\right)^{2}\right]
$$

with scalar and pseudoscalar interactions corresponding to $\sigma$ and $\pi$ excitations respectively, where $m_{0}$ is the current quark mass, $G$ is the four-quark coupling constant with dimension $(\mathrm{GeV})^{-2}, \tau_{i}(i=1,2,3)$ are the Pauli matrices in flavor space, and $\mu$ is the quark chemical potential which is one third of the baryon chemical potential $\mu_{B}$.

In mean field approximation the inverse quark propagator can be written as

$$
S^{-1}(P)=\gamma^{\mu} P_{\mu}-M+\gamma^{0} \mu
$$

where the dynamically generated quark mass $M$ in the medium is related to the order parameter $\langle\bar{\psi} \psi\rangle$ of the chiral phase transition through $M=m_{0}-2 G\langle\bar{\psi} \psi\rangle$ and is determined by the self-consistent gap equation,

$$
M=m_{0}+2 i G \int \frac{d^{4} P}{(2 \pi)^{4}} \operatorname{Tr}[S(P)],
$$

where and in the following the trace is taken in color, flavor and Dirac spaces. After the summation over Matsubara fermion frequencies in the imaginary formalism of finite temperature field theory, the gap equation can be reexpressed as

$$
M\left(1-2 G I_{1}\right)=m_{0}
$$

Since the NJL model is non-renormalizable, one should take a regularization scheme to avoid the divergence in the momentum integration (44). By taking into account the covariant Pauli-Villars regularization approach, we can separate the function $I_{1}$ in (5) into a vacuum and a matter part [15],

$$
\begin{aligned}
& I_{1}=I_{1}^{v a c}+I_{1}^{\text {mat }} \\
& I_{1}^{v a c}=\frac{3}{2 \pi^{2}} \sum_{j=0}^{N} A_{j} \Lambda_{j}^{2} \ln \Lambda_{j}^{2} \\
& I_{1}^{\text {mat }}=-\frac{6}{\pi^{2}} \sum_{j=0}^{N} \sum_{a= \pm} A_{j} \int_{0}^{\infty} d p \frac{p^{2}}{E_{j}(p)} f\left(E_{j}^{a}(p)\right)
\end{aligned}
$$

with the definition of $E_{j}^{ \pm}(p)=E_{j}(p) \pm \mu$ and $E_{j}(p)=$ $\sqrt{p^{2}+\Lambda_{j}^{2}}$, the Fermi-Dirac distribution function $f(x)=$ $1 /\left(e^{x / T}+1\right)$ at finite temperature $T$, and the number of subtractions $N$. In the following numerical calculations we take the same parameter values as used in Ref. [15], $m_{0}=8.56 \mathrm{MeV}, G=0.75 / 2 \mathrm{fm}^{2}, N=3, \Lambda_{0}=M$, $\Lambda_{1}=680 \mathrm{MeV}, \Lambda_{2}=2.1 \Lambda_{1}, \Lambda_{3}=2.1 \Lambda_{2}$ and $A_{0}=1$, and $A_{1}, A_{2}, A_{3}$ are determined by the constraints $\sum_{j=0}^{N} A_{j}=$ $0, \sum_{j=0}^{N} A_{j} \Lambda_{j}^{2}=0$ and $\sum_{j=0}^{N} A_{j} \Lambda_{j}^{4}=0$. With these values one can fit the pion mass $M_{\pi}=138 \mathrm{MeV}$ and pion decay constant $f_{\pi}=94 \mathrm{MeV}$ and fix the quark mass $M=376 \mathrm{MeV}$ and sigma mass $M_{\sigma}=760 \mathrm{MeV}$ in the vacuum.

In the NJL model, mesons are considered as quantum fluctuations above the mean field and can be constructed in random phase approximation (RPA). After the bubble summation in RPA, the meson propagator is expressed as 12,14

$$
U_{m}\left(k_{0}^{2}, k^{2}\right)=\frac{-2 G}{1-2 G \Pi_{m}\left(k_{0}^{2}, k^{2}\right)}
$$

with the meson polarization functions

$$
\Pi_{m}\left(k_{0}^{2}, k^{2}\right)=i \int \frac{d^{4} P}{(2 \pi)^{4}} \operatorname{Tr}\left[\Gamma_{m}^{*} S\left(P+\frac{K}{2}\right) \Gamma_{n} S\left(P-\frac{K}{2}\right)\right],
$$

and the meson vertexes

$$
\Gamma_{m}=\left\{\begin{array}{ll}
1 & m=\sigma \\
i \tau_{+} \gamma_{5} & m=\pi_{+} \\
i \tau_{-} \gamma_{5} & m=\pi_{-} \\
i \tau_{3} \gamma_{5} & m=\pi_{0}
\end{array} \quad \Gamma_{m}^{*}= \begin{cases}1 & m=\sigma \\
i \tau_{-} \gamma_{5} & m=\pi_{+} \\
i \tau_{+} \gamma_{5} & m=\pi_{-} \\
i \tau_{3} \gamma_{5} & m=\pi_{0}\end{cases}\right.
$$

Since the Lorentz invariance is explicitly broken at finite temperature, the polarization functions depend on $k_{0}^{2}$ and $\mathbf{k}^{2}$ separately.

With the NJL meson propagator (7), the quark potential through a meson exchange is expressed as

$$
\begin{aligned}
V_{m}(r) & =\int \frac{d^{3} \mathbf{k}}{(2 \pi)^{3}} e^{i \mathbf{k} \cdot \mathbf{r}} U_{m}\left(0, k^{2}\right) \\
& =-\frac{G}{2 \pi^{2} r} \operatorname{Im} \int_{-\infty}^{\infty} d k \frac{k e^{i k r}}{1-2 G \Pi_{m}\left(0, k^{2}\right)}
\end{aligned}
$$

By constructing a contour in the complex meson momentum plane, the above integration can be done via calculating the meson pole in the complex plane. To this end, we need to know the analytic structure of the meson polarization function $\Pi_{m}\left(k_{0}^{2}, k^{2}\right)$.

In the Pauli-Villars regularization scheme we again divide the polarization function into a vacuum and a matter part,

$$
\Pi_{m}\left(k_{0}^{2}, k^{2}\right)=\Pi_{m}^{v a c}\left(k_{0}^{2}, k^{2}\right)+\Pi_{m}^{m a t}\left(k_{0}^{2}, k^{2}\right) .
$$

From the discontinuity of the matter part on the real axis and the discontinuity of the vacuum part on the imaginary axis [15], 


$$
\begin{aligned}
& \operatorname{Re} \Pi_{m}^{v a c}\left(0,(k \pm i \epsilon)^{2}\right)=I_{1}^{v a c}+\frac{3\left(k^{2}+\epsilon_{m}^{2}\right)}{2 \pi^{2}} \sum_{j=0}^{N} A_{j}\left[\frac{1}{k_{j}} \sqrt{1+k_{j}^{2}} \ln \left(\sqrt{1+k_{j}^{2}}+k_{j}\right)+\ln \Lambda_{j}\right] \\
& \operatorname{Re} \Pi_{m}^{m a t}\left(0,(k \pm i \epsilon)^{2}\right)=I_{1}^{m a t}-\frac{3\left(k^{2}+\epsilon_{m}^{2}\right)}{2 \pi^{2} k} \sum_{j=0}^{N} \sum_{a= \pm} A_{j} \int_{0}^{\infty} d p \frac{p}{E_{j}(p)} \ln \left|\frac{k-2 p}{k+2 p}\right| f\left(E_{j}^{a}(p)\right), \\
& \operatorname{Im} \Pi_{m}^{v a c}\left(0,(k \pm i \epsilon)^{2}\right)=0 \\
& \operatorname{Im} \Pi_{m}^{m a t}\left(0,(k \pm i \epsilon)^{2}\right)=\mp \frac{3 T\left(k^{2}+\epsilon_{m}^{2}\right)}{4 k \pi} \sum_{j=0}^{N} \sum_{a= \pm} A_{j} \ln \left(1+e^{-E_{j}^{a}(k / 2)}\right), \\
& \operatorname{Re} \Pi_{m}^{v a c}\left(0,(i k \pm \epsilon)^{2}\right)= \begin{cases}I_{1}^{v a c}+\frac{3\left(\epsilon_{m}^{2}-k^{2}\right)}{2 \pi^{2}} \sum_{j=0}^{N} A_{j}\left[\frac{1}{k_{j}} \sqrt{1-k_{j}^{2}} \arcsin k_{j}+\ln \Lambda_{j}\right] & \text { for } k_{j}<1 \\
I_{1}^{v a c}+\frac{3\left(\epsilon_{m}^{2}-k^{2}\right)}{2 \pi^{2}} \sum_{j=0}^{N} A_{j}\left[\frac{1}{k_{j}} \sqrt{k_{j}^{2}-1} \ln \left(k_{j}+\sqrt{k_{j}^{2}-1}\right)+\ln \Lambda_{j}\right] & \text { for } k_{j}>1\end{cases} \\
& \operatorname{Re} \Pi_{m}^{m a t}\left(0,(i k \pm \epsilon)^{2}\right)=I_{1}^{m a t}-\frac{3\left(\epsilon_{m}^{2}-k^{2}\right)}{2 \pi^{2} k} \sum_{j=0}^{N} \sum_{a= \pm} A_{j} \int_{0}^{\infty} d p \frac{p}{E_{j}(p)}\left[\pi-2 \arctan \left(\frac{k}{2 p}\right)\right] f\left(E_{j}^{a}(p)\right) \text {, } \\
& \operatorname{Im} \Pi_{m}^{v a c}\left(0,(i k \pm \epsilon)^{2}\right)= \begin{cases}0 & \text { for } k_{j}<1 \\
\pm \frac{3\left(\epsilon_{m}^{2}-k^{2}\right)}{4 \pi} \sum_{j=0}^{N} A_{j} \frac{1}{k_{j}} \sqrt{k_{j}^{2}-1} & \text { for } k_{j}>1\end{cases} \\
& \operatorname{Im} \Pi_{m}^{m a t}\left(0,(i k \pm \epsilon)^{2}\right)=0
\end{aligned}
$$

with the definition of $k_{j}=k /\left(2 \Lambda_{j}\right)$ and $\epsilon_{m}^{2}=0$ for $m=$ $\pi_{+}, \pi_{-}, \pi_{0}$ and $\epsilon_{m}^{2}=4 M^{2}$ for $m=\sigma$, there is a vacuum cut on the imaginary axis starting at $2 m$ and a matter cut on the real axis in the complex meson momentum plane. To replace the direct momentum integration (10) by the contour integration in the complex momentum plane, we need to know the pole position of the meson propagator. Suppose the pole is at $k=i M_{m}+\Gamma_{m}$, the mass $M_{m}$ and width $\Gamma_{m}$ are determined by the complex pole equation

$$
1-2 G \Pi_{m}\left(0,\left(i M_{m}+\Gamma_{m}\right)^{2}\right)=0 .
$$

Since the Lorentz invariance is broken at finite temperature, the mass $M_{m}$ and width $\Gamma_{m}$ related to the quark potential are in general different from the dynamic meson mass $\bar{M}_{m}$ and the corresponding width $\bar{\Gamma}_{m}$ controlled by the pole equation

$$
1-2 G \Pi_{m}\left(\left(\bar{M}_{m}-i \bar{\Gamma}_{m}\right)^{2}, 0\right)=0 .
$$

Only in the vacuum with $T=\mu=0$, the two poles coincide.

Suppose the width is much less than the mass, $\Gamma_{m}<<$ $M_{m}$, the mass is decoupled from $\Gamma_{m}$,

$$
1-2 G \operatorname{Re} \Pi_{m}\left(0,\left(i M_{m}+\epsilon\right)^{2}\right)=0,
$$

and $\Gamma_{m}$ is determined by

$$
\Gamma_{m}=-\frac{\epsilon_{m}^{2}-M_{m}^{2}}{2 M_{m}} \frac{\operatorname{Im} \Pi_{m}\left(0,\left(i M_{m}+\epsilon\right)^{2}\right)}{\operatorname{Re} \Pi_{m}\left(0,\left(i M_{m}+\epsilon\right)^{2}\right)-I_{1}} .
$$

Due to the mass constraint $\bar{M}_{\pi}<2 M$ at $T<T_{c}$, pions are bound states and can not decay into two quarks, and therefore they have no width in the chiral breaking phase. In chiral limit with zero current quark mass, from the mass relations $M_{m}=\epsilon_{m}$ at $T<T_{c}$, not only pions but also sigma have no widths in the chiral breaking phase.

Taking into account the contributions from the vacuum cut, matter cut and the pole to the total quark potential, we have

$$
V_{m}(r)=V_{m}^{v a c}(r)+V_{m}^{m a t}(r)+V_{m}^{p o l}(r)
$$

with the vacuum part

$$
\begin{aligned}
V_{m}^{\text {vac }}(r)= & \frac{G}{2 \pi^{2} r} \int_{2 M}^{\infty} d k k e^{-k r} \operatorname{Im}\left[\frac{1}{1-2 G \Pi_{m}\left(0,(i k+\epsilon)^{2}\right)}\right. \\
& \left.-\frac{1}{1-2 G \Pi_{m}\left(0,(i k-\epsilon)^{2}\right)}\right]
\end{aligned}
$$

which decays exponentially, the matter part

$$
\begin{aligned}
V_{m}^{m a t}(r)= & -\frac{G}{2 \pi^{2} r} \int_{-\infty}^{\infty} d k k \operatorname{Im} e^{i k r}\left[\frac{1}{1-2 G \Pi_{m}\left(0, k^{2}\right)}\right. \\
& \left.-\frac{1}{1-2 G \Pi_{m}\left(0,(k+i \epsilon)^{2}\right)}\right]
\end{aligned}
$$

which in general oscillates, and the pole part

$$
V_{m}^{p o l}(r)=\frac{1}{2 \pi} \frac{e^{-M_{m} r}}{r} \frac{a_{m} \cos \left(\Gamma_{m} r\right)+b_{m} \sin \left(\Gamma_{m} r\right)}{a_{m}^{2}+b_{m}^{2}}
$$

with the coefficients $a_{m}$ and $b_{m}$ defined by

$$
\left.\frac{\partial \Pi_{m}\left(0, k^{2}\right)}{\partial k^{2}}\right|_{k=\Gamma_{m}+i M_{m}}=a_{m}+i b_{m},
$$


where the mass and width control, respectively, the amplitude and the oscillation frequency of the pole contribution. In the chiral breaking phase, the pole is exactly on the imaginary axis, we recover the traditional Yukawa form

$$
V_{m}^{p o l}(r)=\frac{1}{2 \pi} \frac{e^{-M_{m} r}}{a_{m} r}
$$

\section{RESULTS}

We first show the effect of chiral symmetry restoration on the quark potential in hot and dense medium. With the parameter values in the model and in the PauliVillars regularization scheme, shown in the last section, the pole mass $M_{m}$ and width $\Gamma_{m}$ as functions of temperature at zero chemical potential are presented in Fig 1 for $m=\pi, \sigma$. In chiral limit, the critical temperature $T_{c}$ for chiral phase transition is about $300 \mathrm{MeV}$. With different parameter values or choosing other regularization schemes, the critical temperature can be reduced. For instance, in the three-momentum non-covariant cutoff scheme, the critical value is $T_{c}=170 \mathrm{MeV}$ [14]. In the real world with nonzero current quark mass, pions and sigma behave differently in the chiral breaking phase. Pions are bound states with zero width, but sigma mass is slightly larger than two times the quark mass. Therefore, there is a small width with sigma at low temperature. When chiral symmetry is restored at high temperature $T>T_{c}$, the difference between pions and sigma disappears, both the masses and widths coincide. The width is always much smaller than the corresponding mass. Even at high temperature $T / T_{c}=3$ the ratio $\Gamma_{m} / M_{m}$ is only about $10 \%$. This is in consistent with the assumption of $\Gamma_{m}<<M_{m}$ for the decoupling of $M_{m}$ from $\Gamma_{m}$, (15) and (16). The meson masses get saturated when the temperature is high enough, this will lead to the saturation of the quark potential, see the following discussion. Note that, the finite width plays a crucial rule in the chiral restoration phase. At $T>T_{c}$, quarks become almost massless, and pions and sigma satisfy the decay condition $M_{m}>2 M$. If the meson width is neglected, the pole is located on the imaginary axis and goes into the vacuum cut, and the pole contribution will be fully suppressed. However, for a nonzero width, even if it is very small compared with the mass, the pole position deviates from the imaginary axis, it is outside the vacuum cut and its contribution is not removed from the quark potential.

To directly see the effect of chiral symmetry restoration on the quark potential, we show in Fig2 2 the pionmediated potentials at $T / T_{c}=1.3$ and $\mu=0$ in the cases of with and without chiral symmetry restoration, and compare them with the potential in the vacuum $(T=\mu=0)$. For the calculation without chiral phase transition, we keep the quark mass and pion mass as their values in the vacuum and remain only the explicit temperature dependence in the distribution function $f$.
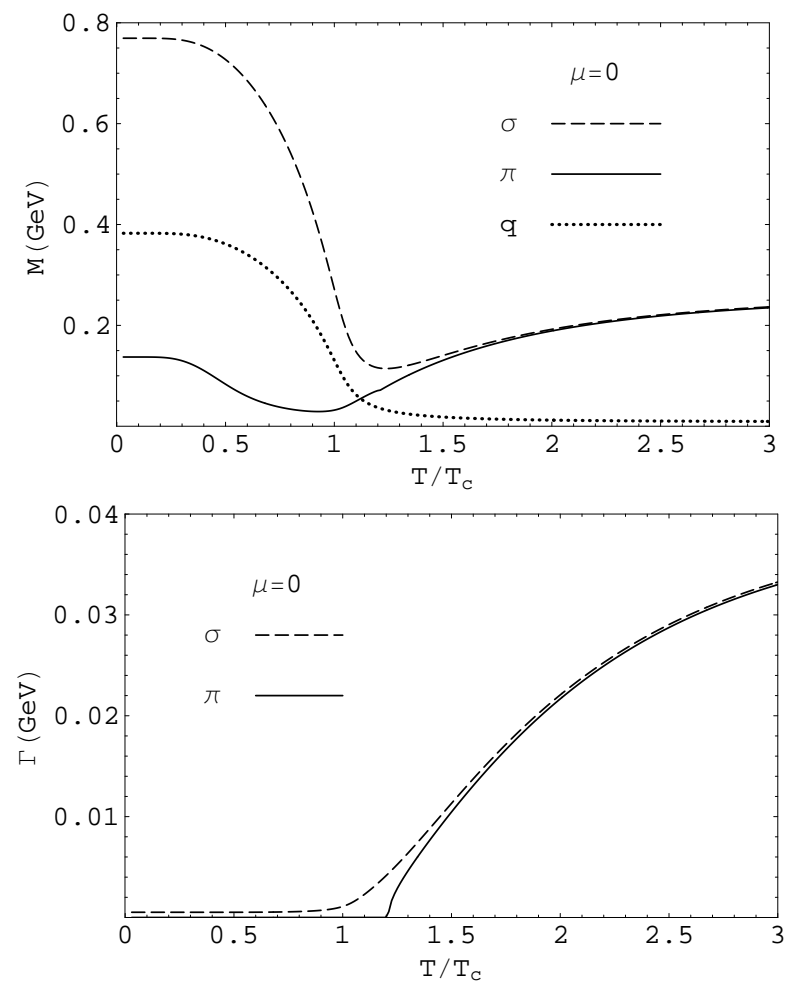

FIG. 1: The quark mass, meson masses (upper panel) and meson widths (lower panel) as functions of temperature at zero chemical potential. The temperature is scaled by the critical value $T_{c}$.

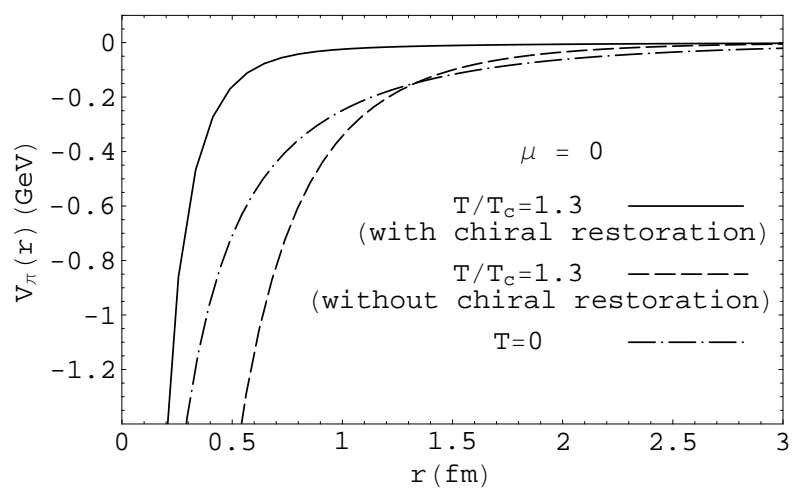

FIG. 2: The pion-mediated quark potentials with and without considering chiral symmetry restoration. The results are compared with the potential in the vacuum.

In this case, the quark potential becomes stronger in comparison with the vacuum potential, because of the increasing quark density in hot medium, and is significantly different from the calculation with chiral symmetry restoration where the potential is much weaker than the vacuum potential, due to the change in the chiral dynamics.

Since sigma is much heavier than pion in the phase 
with chiral symmetry breaking, and they become degenerate when the symmetry is restored, sigma exchange is remarkably reflected in the quark potential only in the short range for $T<T_{c}$ and becomes equivalently important as pion exchange for $T>T_{c}$. This is explicitly shown in Fig 3 ,

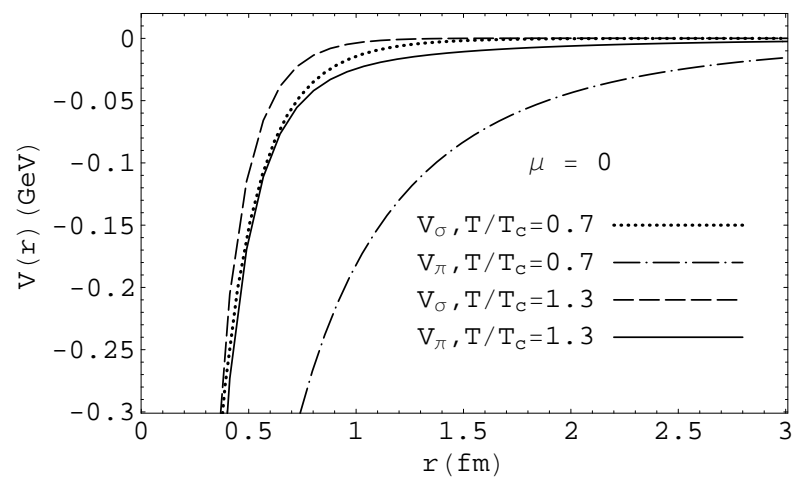

FIG. 3: The pion- and sigma-mediated quark potentials in the chiral breaking and chiral restoration phases.
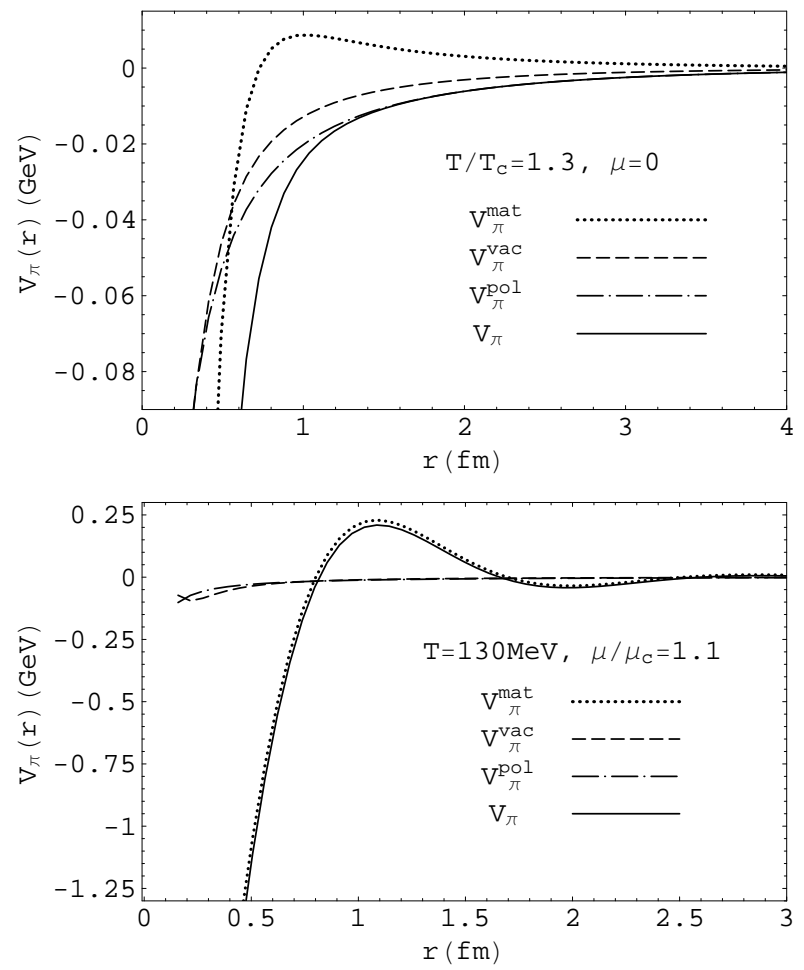

FIG. 4: The pion-mediated quark potential and its three components arising from the vacuum cut, matter cut, and pole in the chiral restoration phase at high temperature $(\mu=0$ and $T / T_{c}=1.2$, upper panel) and high chemical potential ( $T=130 \mathrm{MeV}$ and $\mu / \mu_{c}=1.1$, lower panel).

We now discuss the two possible oscillations in the quark potential, the Friedel oscillation and the Yukawa oscillation. The former is due to the sharp Fermi surface and the latter arises from complex poles of meson exchanges. From the matter and pole contributions (19) and (20), there are always oscillations in the two parts. To know whether they can be clearly reflected in the total potential, we separately show the three contributions from the vacuum cut, matter cut and pole in Fig,4. While there is a remarkable oscillation around $r=1 \mathrm{fm}$ in the matter part at $\mu=0$ and $T / T_{c}=1.3$, see the upper panel, it can not be seen clearly in the total potential, since at high temperature and low density the matter contribution is not the dominant one in the total potential. In this case, only Yukawa oscillation can be seen, if the width is large enough. At $T / T_{c}=1.3$ the pion width is too small and the induced weak oscillation is difficult to be demonstrated in the total potential. On the other hand, at low temperature and high density, the quark potential is absolutely controlled by the matter cut, see the lower panel at $\mu / \mu_{c}=1.1$ with $\mu_{c}=400 \mathrm{MeV}$ being the critical chemical potential for $T=130 \mathrm{MeV}$, and only the Fermi-surface induced Friedel oscillation can be seen in the total potential. When the temperature and chemical potential are not low enough, especially in the chiral restoration phase, the contribution from the vacuum cut is always smaller than the matter part and pole part.

The Friedel oscillation is related to the singularity of the matter part of the meson polarization function $\Pi_{m}$ at $k=2 p_{f}$ on the real axis at zero temperature, where $p_{f}$ is the quark Fermi momentum, see (12). At finite temperature, there is no more strict Fermi surface and singularity in the polarization function, the oscillation will become weak at low temperature and finally disappear at high enough temperature. In the upper panel of Fig 5 we plot the quark potential at fixed chemical potential for three values of temperature. The Friedel oscillation is gradually washed away by increasing temperature. The chemical potential dependence of the Friedel oscillation at fixed temperature is shown in the lower panel. In the chiral breaking phase with low chemical potential $\mu<M$, there is no Fermi momentum and in turn no Friedel oscillation. The oscillation happens only in the chiral restoration phase, and the amplitude and frequency increase with increasing chemical potential.

The strength of the Yukawa oscillation depends strongly on the pole width. In Fig 6 we show the pionmediated quark potential in the chiral restoration phase with $\mu=0$ and $T / T_{c}=2$ for three different values of width. As we emphasized above, the potential at high temperature and low density is dominated by the pole contribution. From the analytic structure of the meson polarization function (12), its matter part is continuous on the imaginary axis, and only the vacuum part contributes to the width (16). From our numerical calculation, see Fig 1 the width is only about $10 \%$ of the mass at $T / T_{c}=2$, and therefore the Yukawa oscillation can not be clearly shown in Fig 6. However, this small width can not be neglected, otherwise the pole will be located on the imaginary axis and go into the vacuum cut, and has 

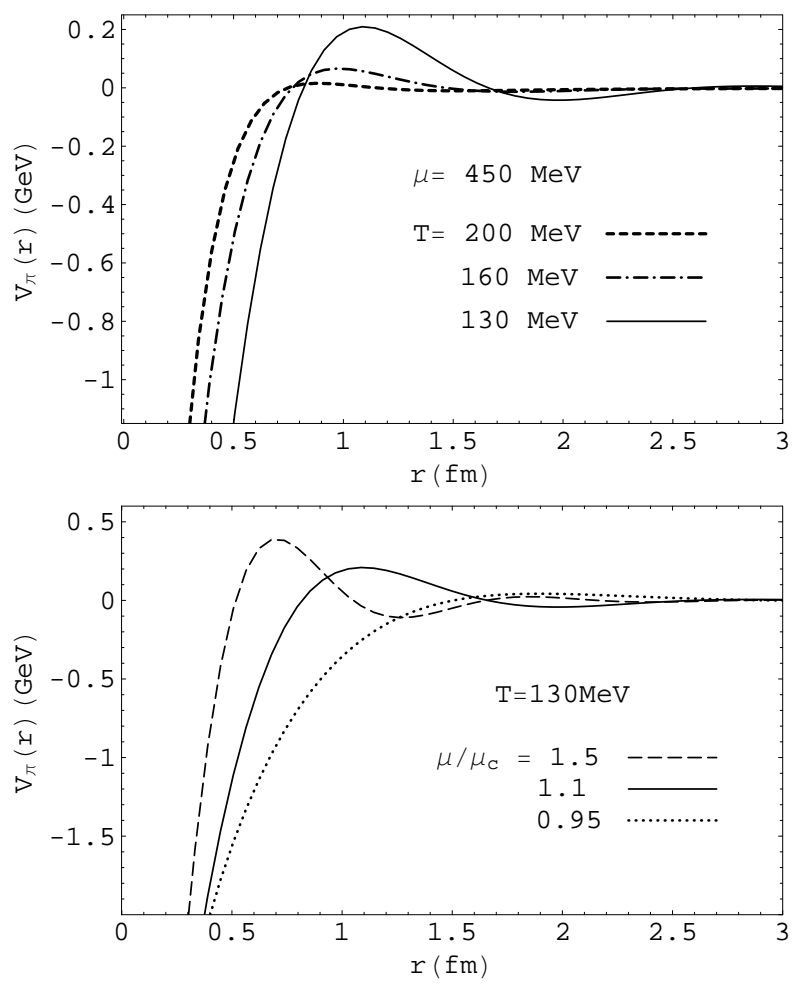

FIG. 5: The temperature (upper panel) and chemical potential (lower panel) dependence of the Friedel oscillation in the pion-mediated quark potential.

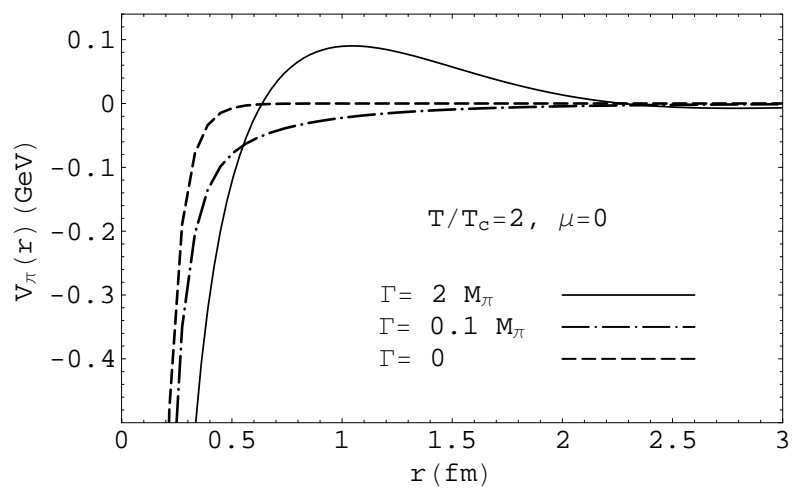

FIG. 6: The width dependence of the Yukawa oscillation in the pion-mediated quark potential in the chiral restoration phase.

no contribution to the potential. To see a clear Yukawa oscillation, we take artificially the width as a free parameter and calculate the potential with $\Gamma_{\pi} / M_{\pi}=2$. In this case, there is really a strong oscillation around $r=1 \mathrm{fm}$, as we expected.

From the analysis of the experimental data obtained in relativistic heavy ion collisions, especially the anisotropic flow, and the lattice and model calculation on the resonant states of quarks and gluons above the critical tem- perature, it is widely accepted that the parton system above and close to the critical temperature is a strongly coupled quark-gluon plasma. The temperature region is about $1 \leq T / T_{c}<3 \sim 4$ [21]. Above this region, quarks and gluons are weakly coupled and the system can approximately be considered as an ideal gas. In Fig 7 we show the temperature dependence of the pion-mediated quark potential at zero chemical potential. With increasing temperature, the potential becomes weaker and weaker due to the chiral symmetry restoration, and finally gets saturated for $T / T_{c} \gtrsim 3$. Since the used PauliVillars regularization scheme guarantees the ideal gas limit at $T \rightarrow \infty$ [15], we can conclude from this saturation that, the quark-meson plasma is strongly coupled in the temperature region $1 \leq T / T_{c} \lesssim 3$. Above $T / T_{c} \simeq 3$, the system behaves like an ideal gas. While there are no gluons and confinement in the NJL model, the quarkmeson plasma has the similar strong coupling region as the quark-gluon plasma.

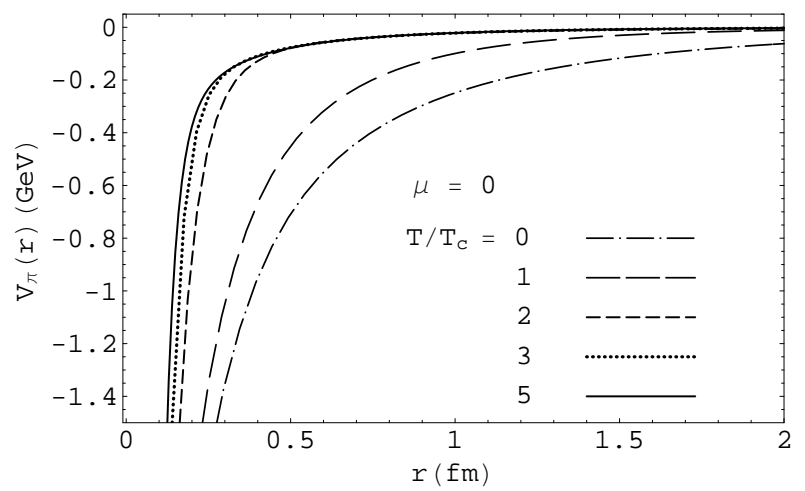

FIG. 7: The temperature dependence of the pion-mediated quark potential at zero chemical potential. The potential gets saturated at $T / T_{c}=3$.

To describe the degree of particle coupling in matter, one can introduce the ratio of particle potential to particle kinetic energy [22],

$$
\gamma=V_{0} / E_{k i n} .
$$

A large ratio means a strongly coupled matter and a small ratio indicates a weakly coupled matter. For the considered quark-meson plasma, we take the potential $V_{0}$ as the meson-mediated quark potential at the average distance between two quarks,

$$
V_{0}=\left|V\left(2 r_{0}\right)\right|,
$$

where $V$ is the summation of $V_{m}$ over all possible meson exchanges, the averaged quark radius $r_{0}$ is determined by the quark and anti-quark number densities $n_{q}$ and $n_{\bar{q}}$,

$$
\begin{aligned}
& \left(n_{q}+n_{\bar{q}}\right) \frac{4}{3} \pi r_{0}^{3}=1, \\
& n_{q, \bar{q}}(T, \mu)=\int \frac{d^{3} \mathbf{p}}{(2 \pi)^{3}} f(E(p) \mp \mu),
\end{aligned}
$$


with $E(p)=\sqrt{\mathbf{p}^{2}+M^{2}}$, and the quark kinetic energy is

$$
\begin{aligned}
E_{k i n}= & \frac{1}{n_{q}+n_{\bar{q}}} \int \frac{d^{3} \mathbf{p}}{(2 \pi)^{3}}(E(p)-M) \\
& \times(f(E(p)-\mu)+f(E(p)+\mu)) .
\end{aligned}
$$

The ratio is shown in Fig 8 as a function of chemical potential at fixed temperature $T=130 \mathrm{MeV}$. Different from the kinetic energy $E_{k i n}$ which monotonously goes up with increasing temperature or chemical potential, the potential $V_{0}$ depends on both the shape of the potential and the quark density. The former is controlled by chiral dynamics and the latter is determined by thermodynamics. With increasing temperature or chemical potential, the quark potential becomes weak, while the density increases and in turn the radius $r_{0}$ gets small. From our numerical calculation, the ratio is rather small at low and high chemical potentials, but peaks strongly at the critical point. This tells us that the strongest coupling in the quark-meson plasma is located at the chiral phase transition. The smoothly increasing ratio after the phase transition is due to the strong Friedel oscillation at high density. From the AdS/CFT estimation 23] and perturbative QCD calculation [24], it is well-known that the ratio of shear viscosity to entropy $\eta / s$ has the minimum value at the critical point of any phase transition, which is widely considered as a general property of strongly coupled matter. The maximum of the ratio $V_{0} / E_{k i n}$ at the chiral phase transition agrees well with the minimum of the ratio $\eta / s$, both describe the strongest coupling at the phase transition.

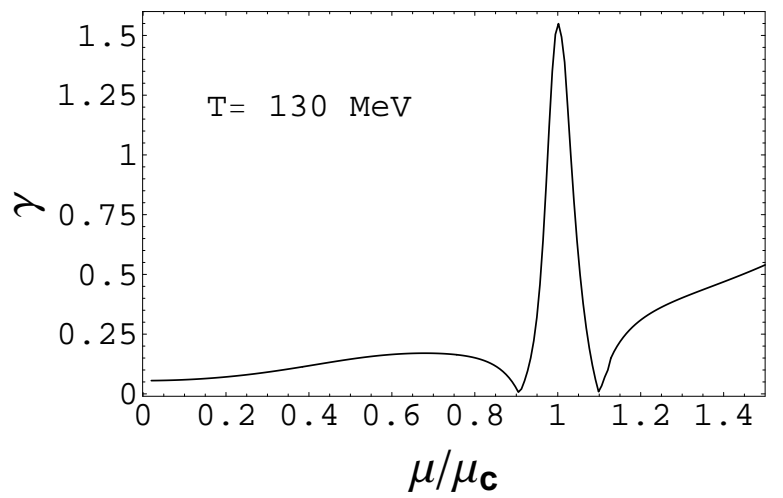

FIG. 8: The ratio of quark potential to quark kinetic energy as a function of chemical potential at $T=130 \mathrm{MeV}$. The peak is located at the critical point of chiral symmetry restoration.

\section{CONCLUSIONS}

In the frame of the two flavor SU(2) NJL model, we calculated the light quark potential through pion and sigma exchanges in RPA approximation. The chiral symmetry restoration at high temperature and density plays an essential rule in the quark potential. While the matter part is always associated with an oscillation, it dominates the potential only for dense matter, and therefore the oscillation can be reflected clearly in the total quark potential only at low temperate and high density. The other possible oscillation in the chiral restoration phase is the Yukawa oscillation induced by the complex poles of mesons. From our self-consistent calculation for meson mass and width, the Yukawa oscillation is weak even at extremely high temperature. The potential gets saturated when temperature is larger than about three times the critical temperature. This means that the strongly coupled quark-meson plasma is in the region of $1 \leq T / T_{c} \lesssim 3$, which agrees well with the widely accepted result for the strongly coupled quark-gluon plasma. Corresponding to the well-known minimum of the ratio of shear viscosity to entropy, we found that the ratio of potential to kinetic energy peaks strongly at the critical point of chiral phase transition.

While we found the strong coupling region for the quark-meson plasma through considering the saturation of quark potential, the saturation temperature $T / T_{c}=3$ looks beyond the effective range of the NJL model. The reliable conclusion is probably that the quark-meson plasma above and close to the chiral critical point is strongly coupled. In our calculation we did not include the vector meson exchange which is expected to contribute a repulsive part to the quark potential and then reduce the depth of the potential well in the short range. To have a complete calculation of the quark potential in the NJL model, one should consider the vector mesons. Another point is the deconfinement effect, it will be helpful to investigate the quark potential using the improved NJL model with Polyakov loop dynamics [19, 20]. Since the original NJL model with pions and sigma describes well the chiral dynamics in the vacuum and at finite temperature and density, we hope our result on the quark potential in the quark-meson plasma is helpful to understand the effect of chiral symmetry restoration on the strongly coupled quark-gluon plasma.

Acknowledgements: We have benefited from discussions with W. Florkowski. P.Z. thanks the Yukawa Institute for Theoretical Physics at Kyoto University and the organizers of NFQCD2008 for their hospitality and for providing the atmosphere of stimulating discussions. The work is supported by the NSFC Grants 10575058 and 10735040 and the 973-project $2007 \mathrm{CB} 815000$. 
[1] M.Gyulassy, nucl-th/0403032, E.Shuryak, J. Phys. G30, S1221(2004).

[2] O.Kaczmarek, S.Ejiri, F.Karsch, E.Laermann and F.Zantow, Prog. Theor. Phys. Suppl. 153, 287(2004).

[3] E.Shuryak and I.Zahed, Phys. Rev. C70, 054507(2004).

[4] J.Kapusta and T.Toimela, Phys. Rev. D37, 3731(1988).

[5] J.Diaz-Aloso, A.Perez Canyellas and H.Sivak, Nucl. Phys. A505, 695(1989).

[6] J.Durso, H.Kim and J.Wambach, Phys. Lett. B298,267(1993).

[7] J.Diaz-Aloso, E.Gallego and A.Perez, Phys. Rev. Lett.73, 2536(1994).

[8] H.Sivak, A.Perez and J.Diaz-Alonso, Prog. Ther. Phys. 105, 961(2001).

[9] E.Epelbaum, Prog. Part. Nucl. Phys. 57, 654(2006).

[10] V.Flambaum and E.Shuryak, Phys. Rev. C76,065206(2007).

[11] Y.Nambu and G.Jona-Lasinio, Phys. Rev. 122 (1961) $345 ; 24$ (1961) 246.

[12] For reviews, see U.Vogl and W.Weise, Prog. Part. and Nucl. Phys. 27 (1991) 195; S.P.Klevansky, Rev. Mod. Phys. 64 (1992) 649; M.K.Volkov, Phys. Part. Nucl. 24 (1993) 35; T.Hatsuda and T.Kunihiro, Phys. Rep. 247 (1994)338; M.Buballa, Phys. Rept. 407, 205(2005).

[13] T.Hatsuda and T.Kunihiro, Phys. Rev. Lett. 55, 158(1985).

[14] J.Hufner, S.Klevansky and P.Zhuang, Ann. Phys. (N. Y.) 234,225(1994); P.Zhuang, J.Hufner and S.Klevansky, Nucl. Phys. A576, 525(1994).

[15] W.Florkowski and B.Friman, Z. Phys. A347, 271(1994) and Acta. Phys. Polon. B25, 49(1994), W.Florkowski, Acta. Phys. Polon. B28,2079(1997).

[16] W.Florkowski and B.Friman, Nucl. Phys. A611, 409(1996).

[17] T.M.Schwarz, S.P.Klevansky and G.Rapp, Phys. Rev.C60, 055205(1999), M.Huang, P.Zhuang and W.Chao, Phys. Rev.D65, 076012(2002), I.Shovkovy and M.Huang, Phys. Lett.B564, 205(2003), D.Ebert, K.G.Klimenko, V.L.Yudichev, Phys. Rev. C72, 015201(2005), S.B.Ruster, V.Werth, M.Buballa, I.A.Shovkovy, D.H.Rischke, Phys. Rev. D72, 034004(2005), D.Blaschke, S.Fredriksson, H.Grigorian, A.M.Oztas, F.Sandin, Phys. Rev. D72, 065020(2005).

[18] M.Frank, M.Buballa and M.Oertel, Phys. Lett. B562, 221(2003), A.Barducci, R.Casalbuoni, G.Pettini, L.Ravagli, Phys. Rev. D71, 016011(2005), L.He, M.Jin and P.Zhuang, Phys. Rev. D71, 116001(2005).

[19] K.Fukushima, Phys. Lett. B591, 277(2004).

[20] C.Ratti, M.A.Thaler and W.Weise, Phys. Rev. D73, 014019(2006).

[21] E.Shuryak and I.Zahed, Phys. Rev. C70, 021901(R)(2004).

[22] B.Gelman, E.Shuryak and I.Zahed, Phys. Rev. C74,044908(2006).

[23] P.Kovtun, D.T.Son, and A.O.Starinets, Phys. Rev. Lett. 94, 111601(2005).

[24] L.P.Csernai, J.I.Kapusta, and L.D.Mclerran, Phys. Rev. Lett. 97, 152303(2006). 